\title{
Overuse of Troponin? A Comprehensive Evaluation of Testing in a Large Hospital System
}

\author{
Gibbs Wilson, MD¹, Kyler Barkley, MD², Kipp Slicker, DO², Robert Kowal, MD, $\mathrm{PhD}^{3}$, \\ Brandon Pope, $\mathrm{PhD}^{3}$, Jeffrey Michel, MD, FACC ${ }^{2 \star}$
}

${ }^{1}$ Department of Internal Medicine and ${ }^{2}$ Department of Cardiology, Scott and White Memorial Hospital, Temple, Texas; ${ }^{3}$ Baylor Scott and White Healthcare, Dallas, Texas.

\begin{abstract}
Troponin assays are integral to the diagnosis of acute myocardial infarction (AMI), but there is concern that testing is over utilized and may not conform to published guidelines. We reviewed all testing performed at 14 hospitals over 12 months and associated troponin values with the primary and secondary diagnoses for each visit. Troponin was determined to be negative, indeterminate or elevated based on reference ranges. The majority of troponin measurements were single, not serial $(64 \%)$. The rate of AMI was low, with only $3.5 \%$ of tested patients having a primary or secondary diagnosis of AMI. Sen-
\end{abstract}

sitivity, specificity and negative predictive value were excellent, exceeding $90 \%$. However, positive predictive value was low, suggesting testing of populations with diseases known to be associated with elevated troponin levels in the absence of AMI. The majority (79\%) of elevated troponin values were associated with primary diagnoses other than AMI. Only $28 \%$ of elevated troponins were associated with a primary or secondary diagnosis of AMI. These data suggest possible overuse of troponin testing in our healthcare system. Journal of Hospital Medicine 2017;12:329-331. (C) 2017 Society of Hospital Medicine
The ability of serum troponin measurement in the diagnosis of acute myocardial infarction (AMI) was validated in patients with at least a moderate pretest probability for the disease. ${ }^{1}$ The diagnostic yield of troponin testing in clinical trials has been between $20 \%$ and $50 \%$, excluded patients thought unlikely to have AMI. In practice, physicians often encounter low-risk patients and patients in whom the diagnosis on initial presentation is unclear. Several noncardiac diagnoses, such as pneumonia and respiratory failure, are associated with an elevated troponin level in the absence of AMI, but patients can present with symptoms similar or identical to those of patients who present with AMI. ${ }^{2-4}$ Elevated troponin level in sepsis has been associated with worsened prognosis, though there is no evidence that this finding alters management. An American College of Cardiology Foundation opinion published in 2012 expressly recommends against troponin testing in patients with sepsis. ${ }^{4}$

The only guideline-based indication for troponin testing is the diagnosis or exclusion of AMI. ${ }^{5}$ We conducted a comprehensive review of troponin testing in our healthcare system to see whether testing might be used in clinical settings in which AMI was unlikely.

\section{METHODS}

We retrospectively obtained data on all visits to 14 hospitals in an integrated healthcare system in Texas between June

\footnotetext{
*Address for correspondence and reprint requests: Jeffrey Michel, MD, FACC, Department of Cardiology, Scott and White Memorial Hospital, $2410 \mathrm{~S}$ 31st St, MS-33-ST156, Temple, TX 76508; Telephone: 512-568-2494; Fax: 254724-9854; E-mail: jeffrey.michel@bswhealth.org
}

Received: May 12, 2016; Revised: September 11, 2016; Accepted: October 17, 2016

2017 Society of Hospital Medicine DOI 10.12788/jhm.2732
2013 and June 2014. We analyzed data for all hospital encounters during which a troponin assay was ordered and a troponin level reported-including qualitative point-of-care assays and quantitative laboratory troponin I measurements. We identified 93,436 visits. Quantitative measurements were divided into negative $(<0.05 \mathrm{ng} / \mathrm{mL})$, indeterminate $(0.05-0.09 \mathrm{ng} / \mathrm{mL})$, and elevated $(>0.09 \mathrm{ng} / \mathrm{mL})$, based on the reference ranges reported to physicians. We associated troponin levels with ICD-9 (International Classification of Diseases, Ninth Revision) primary and secondary diagnoses, grouping ICD-9 codes 410 (AMI), 411 (other acute or subacute forms of ischemic heart disease [IHD]), 412 (old myocardial infarction), 413 (angina pectoris), and 414 (other forms of chronic IHD) as representing IHD diagnoses.

To further evaluate troponin testing, we constructed 2 contingency matrices (Table). ${ }^{6}$ We included visits for which both primary and secondary diagnoses were available for review and for which quantitative troponin I measurements were available; 92,445 encounters met criteria for inclusion in matrix calculations. In the first matrix (part A of Table), a primary diagnosis of any AMI (ICD-9 code 410) was used as "positive" and all others "negative." In the second matrix (part B of Table), "positive" includes any primary or secondary diagnosis of AMI.

\section{RESULTS}

We identified a total of 93,436 hospital visits associated with troponin testing; 179,239 troponin measurements were associated with these visits (an average of 1.81 per encounter). Of these visits, 59,897 (64.1\%) were associated with a single measurement. Of the 179,239 measurements, 147,051 $(82.1 \%)$ were negative, $21,881(12.1 \%)$ indeterminate, and 10,307 (5.8\%) positive. Primary diagnoses of hypertension, 


\begin{tabular}{lccc}
\hline TABLE. Contingency Matrices Evaluating Elevated Troponin Levels and AMI Diagnoses \\
\hline A. Primary Diagnosis of AMl & AMl & No AMl & Total \\
Troponin level, $\geq 0.1 \mathrm{ng} / \mathrm{mL}$ & 2238 & 8069 & 10,307 \\
Troponin level, $<0.1 \mathrm{ng} / \mathrm{mL}$ & 167 & 81,971 & 82,138 \\
Total & 2405 & 90,040 & 92,445 \\
\hline B. Primary or Secondary Diagnosis of AMl & AMl & No AMl & Total \\
Troponin level, $\geq 0.1 \mathrm{ng} / \mathrm{mL}$ & 2969 & 7338 & 10,307 \\
Troponin level, $<0.1 \mathrm{ng} / \mathrm{mL}$ & 294 & 81,844 & 82,138 \\
Total & 3263 & 89,182 & 92,445 \\
\hline C. Primary vs Any Diagnosis of AMl & Primary & Any & 0.035 \\
Prevalence & 0.026 & $95 \%$ Cl & 0.910 \\
Sensitivity & 0.931 & $0.025-0.027$ & $0.95 \% \mathrm{Cl}$ \\
Specificity & 0.910 & $0.920-0.940$ & $0.034-0.037$ \\
Positive predictive value & 0.217 & $0.909-0.912$ & $0.900-0.920$ \\
Negative predictive value & 0.998 & $0.209-0.225$ & $0.916-0.920$ \\
\hline
\end{tabular}

aElevated troponin levels $(\geq 0.1 \mathrm{ng} / \mathrm{mL}$ ) were associated with either (A) primary diagnoses of AMl or (B) all primary or secondary diagnoses of AMl. (C) Disease prevalence, sensitivity, specificity, and positive and negative predictive values were calculated for each condition; Cls were calculated using the log method. ${ }^{11}$

NOTE: Abbreviations: AMl, acute myocardial infarction; Cl, confidence interval.

dizziness, abdominal pain, anxiety, dehydration, and headache associated with troponin testing comprised 6127 encounters and had no associated elevated troponin levels. Several non-cardiac primary diagnoses were associated with significant numbers of elevated troponin values including septicemia (27\%), acute respiratory failure (28\%), and cerebrovascular accident (10\%). Seventy-six percent of encounters associated with troponin testing had no primary or secondary IHD diagnosis. Only $2 \%$ of 16,941 visits with a primary diagnosis of chest pain were associated with abnormal troponin levels (Figure).

Analysis of contingency matrices revealed AMI prevalence of $2.6 \%$ when primary AMI diagnoses were considered

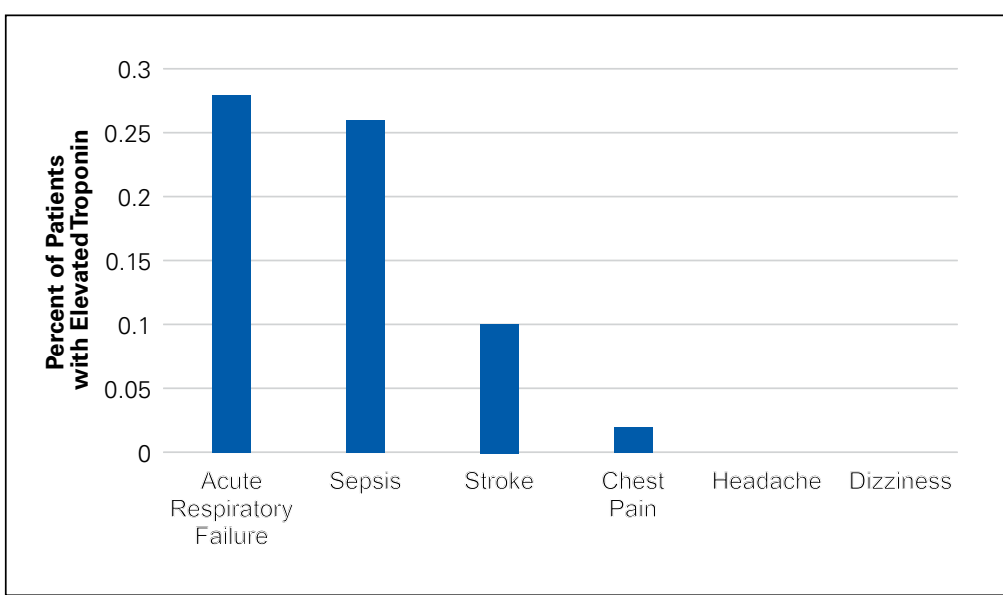

FIG. Notable non-AMI primary diagnoses associated with elevated troponin I values. A selection of primary diagnoses other than $\mathrm{AMI}$ is presented with the corresponding percentage of elevated $(\geq 0.1 \mathrm{ng} / \mathrm{ml})$ troponin I measurements (0.00-1.00). For comparison, the percentage of elevated troponin I measurements associated with a primary diagnosis of AMI was 0.93. NOTE: Abbreviation: AMI, acute myocardial infarction. and 3.5\% when any AMI diagnoses were considered. Sensitivity and specificity were high $(>90 \%)$, and negative predictive value extremely high $(>99 \%)$ in each circumstance. However, positive predictive values were low $(21.7 \%$ and $28.8 \%$, respectively), indicating the majority of patients with elevated troponin levels were not reported to have AMI by attending physicians.

\section{DISCUSSION}

We were surprised to find that troponin level was measured only once during $64 \%$ of the hospital encounters. Although there are clinical scenarios in which a single measurement might be indicated, detecting a rise or fall in troponin level is integral to the diagnosis of AMI, which is why guidelines recommend serial measurement. ${ }^{4}$ We were also surprised to find a low rate of either primary or secondary AMI in patients tested. As others have found, ${ }^{2,3}$ elevated troponin levels were associated with noncardiac primary diagnoses, such as sepsis, respiratory failure, and stroke. Of interest, the majority $(72 \%)$ of patients with elevated troponin levels did not receive a primary or secondary diagnosis of AMI.

Determining the appropriate level of use for a diagnostic laboratory test can be difficult. Primary diagnostic codes, including codes for headache and dizziness, accounted for thousands of tested patients but were associated with no elevated troponin levels. On the other hand, sepsis, pneumonia, and stroke were associated with high rates of elevated troponin levels. Elevated troponin levels likely precipitate cardiology consultation and testing, which increase cost of care perhaps without improving either quality or value of care. Howev- 
er, evidence for the potential prognostic value of testing has led to ongoing research at our institution to evaluate whether troponin measurement might guide better management of such patients.

Appropriate use criteria have been developed for many diagnostic studies, including echocardiography, stress testing, and cardiac catheterization, but not for laboratory testing. Our data suggest possible overuse of troponin testing in our healthcare system. The low AMI incidence we found (2.6\%-3.5\%) indicates that many patients without AMI are being tested.

Although it is impossible to accurately estimate sensitivity and specificity of testing post hoc, it is reassuring to see that measured sensitivity, specificity, and negative predictive values were all high and consistent with published values from prospective clinical trials. ${ }^{7,8}$

As potential roles for troponin testing develop for patients without primary cardiac disease, it becomes even more important to develop guidelines for testing and to avoid universal testing of all hospitalized patients. The high negative predictive value of troponin testing $(99 \%)$ is attractive to physicians who want to avoid missing AMI. Electronic order sets allow troponin testing to be included alongside "standard" testing, such as complete blood cell counts and comprehensive metabolic panels, and may contribute to overuse.

The troponin assays used in our healthcare system in 2014 likely will be replaced with high-sensitivity assays currently being used in Europe. ${ }^{9,10}$ These high-sensitivity assays can improve sensitivity but cannot be expected to increase positive predictive value or reduce false detection rates. When performed as single measurements, hs troponin has the potential to increase the number of elevated troponins detected that are not associated with AMI.

On the basis of our data, we have initiated a system-wide program to improve performance of troponin testing in our healthcare system. We are working with hospitalists and critical care and emergency department physicians to ensure that serial measurements are being performed and that the correct patients are being tested. Future data collection will help determine the success or failure of these efforts.

Disclosure: Nothing to report

\section{References}

1. Thygesen K, Alpert JS, Jaffe AS, et al. Third universal definition of myocardial infarction. Circulation. 2012;126(16):2020-2035.

2. Hamm CW, Giannitsis E, Katus HA. Cardiac troponin elevations in patients without acute coronary syndrome. Circulation. 2002;106(23):2871-2872.

3. Roongsritong C, Warraich I, Bradley C. Common causes of troponin elevations in the absence of acute myocardial infarction: incidence and clinical significance. Chest. 2004;125(5):1877-1884.

4. Newby LK, Jesse RL, Babb JD, et al. ACCF 2012 expert consensus document on practical clinical considerations in the interpretation of troponin elevations: a report of the American College of Cardiology Foundation Task Force on Clinical Consensus Documents. J Am Coll Cardiol. 2012;60(23):2427-2463.

5. Amsterdam EA, Wenger NK, Brindis RG, et al; American College of Cardiology; American Heart Association Task Force on Practice Guidelines; Society for Cardiovascular Angiography and Interventions; Society of Thoracic Surgeons; American Association for Clinical Chemistry. 2014 AHA/ACC Guideline for the Management of Patients With Non-ST-Elevation Acute Coronary Syndromes: a report of the American College of Cardiology/American Heart Association Task Force on Practice Guidelines. J Am Coll Cardiol. 2014;64(24):e139-e228.

6. Pearson K. On the Theory of Contingency and Its Relationship to Association and Normal Correlation. Mathematical Contributions to the Theory of Evolution. London, England: Dulau \& Co; 1904

7. Katus HA, Remppis A, Neumann FJ, et al. Diagnostic efficiency of troponin T measurements in acute myocardial infarction. Circulation. 1991;83(3):902-912.

8. Olatidoye AG, Wu AH, Feng YJ, Waters D. Prognostic role of troponin T versus troponin I in unstable angina pectoris for cardiac events with meta-analysis comparing published studies. Am J Cardiol. 1998;81(12):1405-1410.

9. Reichlin T, Hochholzer W, Bassetti S, et al. Early diagnosis of myocardial infarction with sensitive cardiac troponin assays. N Engl J Med. 2009;361(9):858-867.

10. Keller T, Zeller T, Peetz D, et al. Sensitive troponin I assay in early diagnosis of acute myocardial infarction. N Engl J Med. 2009;361(9):868-877. 